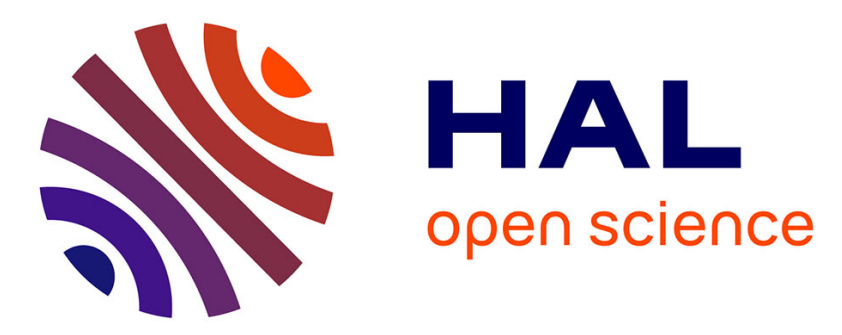

\title{
Natural hyperfine and magnetic predissociation of the I2 B state II. - Experiments on natural and hyperfine predissociation
}

\author{
Jacques Vigué, M. Broyer, J.C. Lehmann
}

\section{- To cite this version: \\ Jacques Vigué, M. Broyer, J.C. Lehmann. Natural hyperfine and magnetic predissociation of the I2 B state II. - Experiments on natural and hyperfine predissociation. Journal de Physique, 1981, 42 (7), pp.949-959. 10.1051/jphys:01981004207094900 . jpa-00209087}

\section{HAL Id: jpa-00209087 https://hal.science/jpa-00209087}

Submitted on 1 Jan 1981

HAL is a multi-disciplinary open access archive for the deposit and dissemination of scientific research documents, whether they are published or not. The documents may come from teaching and research institutions in France or abroad, or from public or private research centers.
L'archive ouverte pluridisciplinaire HAL, est destinée au dépôt et à la diffusion de documents scientifiques de niveau recherche, publiés ou non, émanant des établissements d'enseignement et de recherche français ou étrangers, des laboratoires publics ou privés. 


\title{
Natural hyperfine and magnetic predissociation of the $I_{2} B$ state II. - Experiments on natural and hyperfine predissociation
}

\author{
J. Vigué, M. Broyer and J. C. Lehmann \\ Laboratoire de Spectroscopie Hertzienne de l'E.N.S. $\left({ }^{*}\right), 24$, rue Lhomond, 75231 Paris Cedex 05, France
}

(Reçu le 19 novembre 1980, accepté le 25 mars 1981)

\begin{abstract}
Résumé. - Cet article présente l'ensemble des résultats expérimentaux actuellement disponibles sur la prédissociation naturelle de l'iode; les expériences décrites ne font pas intervenir la prédissociation magnétique. Tous ces résultats sont analysés en tenant compte de la dépendance avec le nombre quantique de rotation de la densité de Franck-Condon de prédissociation. L'accord entre la théorie et l'expérience est bon.
\end{abstract}

\begin{abstract}
This paper presents all presently available experimental results on the natural predissociation of iodine; the experiments described do not involve magnetic predissociation effects. The analysis of all these results takes into account the dependence of the Franck-Condon density on the rotational quantum number. Agreement between theory and experiment is good.
\end{abstract}

1. Introduction. - In a series of letters and papers [1-6], we have presented the main features of the magnetic and natural predissociation effects in the $\mathrm{B}^{3} \Pi_{0^{+} \mathrm{u}}$ state of iodine. In the present paper, we will present a precise numerical analysis of all experiments concerning the natural predissociation of the B state. It will include the presentation of a large number of as yet unpublished experimental results. On the theoretical side, the main contribution of this article will be to include the variation of the Franck-Condon density with rotational quantum number $J$ to interpret the experimental results. From the detailed interpretation of the experiments, a set of predissociation parameters and radiative decay rates will be deduced.

We will begin with a quick historical review of the $I_{2} B$ state natural predissociation, then give a theoretical interpretation of the variation of the Franck-Condon density with $J$ and finally analyse all the experiments involving only natural predissociation. The third paper of this series will analyse magnetic predissociation effects.

2. First experimental results on the natural predissociation of $\mathrm{I}_{2}$ B state. - 2.1 HisTORICAL REVIEW : FIRST THEORY. - In 1967, the hypothesis of natural predissociation of the iodine B state was first formulated by Chutjian et al. [7] in order to interpret the

(*) Associé au C.N.R.S.

LE JOURNAL DE PHYSIQUe - T. 42, No 7, JUILLET 1981 rapid variations of the lifetime, measured for several vibrational levels of the B state. This first experimental evidence was soon confirmed by measurements of absorption line strengths [8]. From these results, the transition moment was deduced and, under some hypothesis, the calculated radiative decay rate was found to be smaller than the observed decay rate for the same levels. From these initial observations, the predissociation process was identified as being of gyroscopic origin and due to a ${ }^{1} \Pi_{1 \mathrm{u}}$ state : the predissociative decay rate was therefore predicted to be given by :

$$
\Gamma_{\text {pred }}(v, J)=C_{v}^{2} J(J+1)
$$

and the total decay rate $\Gamma$ by :

$$
\Gamma=\Gamma_{\mathrm{rad}}(v)+C_{v}^{2} J(J+1)
$$

$\left(\Gamma_{\text {rad }}(v)\right.$ : radiative decay rate).

From the vibrational dependence of $\Gamma_{\text {pred }}$, a first estimate of the potential curve of the ${ }^{1} \Pi_{1 u}$ state was obtained [9]. At the same time, a qualitative manifestation of this predissociation effect was obtained by directly observing the atoms produced by this process $[10,11]$.

Then L. Brewer and J. Tellinghuisen made a complete study of the problem : they measured the photodissociation quantum yield of $I_{2}$ as a function of excitation wavelength [12]. This work was implemented by resolution of the iodine visible absorption spectrum into its discrete $\left(\mathrm{B}^{3} \Pi_{0^{+} \mathrm{u}} \leftarrow \mathrm{X}\right)$ and continuum 
$\left(\mathrm{A}^{3} \Pi_{1 \mathrm{u}} \leftarrow \mathrm{X}\right.$ and $\left.{ }^{1} \Pi_{1 \mathrm{u}} \leftarrow \mathrm{X}\right)$ parts [13], and by several lifetime measurements. From all these data, Tellinghuisen was able to deduce a rather precise vibrational dependence of the predissociation rate [14]. This dependence was in good agreement with the ${ }^{1} \Pi_{1 \mathrm{u}}$ potential curve obtained from the absorption spectrum [13]. The derived $C_{v}^{2}$ curve exhibits two maxima : one around $v=5\left(C_{v}^{2} \approx 1700 \mathrm{~s}^{-1}\right)$ and one around $v=25\left(C_{v}^{2} \approx 450 \mathrm{~s}^{-1}\right)$. It is important to note that the validity of equation (1) was not confirmed experimentally because the experiments [12] did not resolve the rotational structure. As a result, the deduction of $C_{v}^{2}$ was indirect.

2.2 EVIDENCE FOR THE DEFICIENCIES OF THIS THEORY. - A few years ago we observed that, in the presence of a magnetic field, a strong circular polarization of the fluorescence from the $I_{2} B$ state appeared [1]. This was interpreted as an interference effect between natural and magnetic predissociations of the B state. This gave not only proof that the magnetic and natural predissociations are both due to the same ${ }^{1} \Pi_{1 \mathrm{u}}$ state, but also a method for measuring accurately the coefficient $C_{v}^{2}$ [2]. The values of $C_{v}^{2}$ obtained in this way were five to ten times smaller than the evaluation by Tellinghuisen [14].

In order to clarify this discrepancy, it seemed important to systematically measure the decay rate of individual levels as a function of their vibrational and rotational quantum numbers in order to test equation (2). Through this experiment [3, 4], it was possible to confirm the values of $C_{v}^{2}$ obtained by the magnetic predissociation interference effect and to prove the existence of an hyperfine predissociation. The theory of this predissociation is given in paper I[15] and is very briefly summarized in the following paragraph.

2.3 THEORY OF A GYROSCOPIC AND HYPERFINE PREDissociation. - The hyperfine sublevels of the $B$ state are $\left|\mathrm{B}{ }^{3} \Pi_{0^{+} u}, v, J, \varepsilon, F\right\rangle$ with the definition

$$
\begin{aligned}
\left|\mathrm{B}^{3} \Pi_{0^{+} \mathrm{u}} v, J, \varepsilon, F\right\rangle= & \\
& =\sum_{I} \alpha(I \varepsilon J F)\left|\mathrm{B}^{3} \Pi_{0^{+} \mathrm{u}} v, J, I, F\right\rangle
\end{aligned}
$$

where $I$ is the total nuclear spin and $F$ the total angular momentum. The $\alpha$ coefficients can be obtained by diagonalization of the hyperfine Hamiltonian. The predissociation rate due to gyroscopic and hyperfine effects is :

$$
\Gamma_{\text {pred }}(v, J, \varepsilon, \dot{F})=\sum_{I}|\alpha(I \varepsilon J F)|^{2} \Gamma_{I J F}
$$

with :

$$
\begin{aligned}
& \Gamma_{I J F}=C_{v}^{2} J(J+1)+ \\
& +\frac{a_{v}^{2}}{3}\left(\mathbf{I}^{2}+\frac{3(\mathbf{I} . \mathbf{J})^{2}+\frac{3}{2} \mathbf{I} . \mathbf{J}-\mathbf{I}^{2} . \mathbf{J}^{2}}{(2 J-1)(2 J+3)}\right)-a_{v} C_{v} \sqrt{2} \mathbf{I} . \mathbf{J}
\end{aligned}
$$

$$
\mathbf{I . J}=(F(F+1)-J(J+1)-I(I+1)) / 2 .
$$

The interpretation of the three terms in equations (5) is very simple [15] : the $C_{v}^{2}$ term is the pure gyroscopic term, the $a_{v}^{2}$ term is the pure hyperfine term, and the $a_{v} C_{v}$ term is an interference term. We recall that this equation is a simplified form of the general equation [15], which appears to be sufficient to fit the data.

The quantities $a_{v}^{2}, C_{v}^{2}$ and $a_{v} C_{v}$ are proportional to the Franck-Condon density for predissociation, if we neglect the variations with the internuclear distance $r$ of the electronic matrix elements appearing in $a_{v}$ and $C_{v}$ (these variations are due either to explicit factors like the $r^{-2}$ factor appearing in $C_{v}$ or to an implicit variation of the electronic wavefunctions with $r$ ). The variation with $J$ of the Franck-Condon density for predissociation creates an implicit variation with $J$ of these three quantities which adds to the explicit ones appearing in equation (5), and we should replace $a_{v}$ and $C_{v}$ in equation (5) by $a_{v J}$ and $C_{v J}$.

\section{J-dependence of the Franck-Condon density. -} 3.1 Generalities. - It is well known that, due to the modification of the potential curves by the centrifugal term $\frac{\hbar^{2}}{2 \mu r^{2}} J(J+1)$, the nuclear radial wavefunctions and, hence, the Franck-Condon densities are dependent on rotational quantum numbers. This effect is usually assumed to be weak and therefore often neglected. Nonetheless, experimental evidence of its importance has already been obtained in predissociation studies (e.g. OD $\mathrm{A}^{2} \Sigma^{+}$state predissociation [16]).

In the present case the potential curve crossing is on the inner wall of the B state (Fig. 1), i.e. it is a $\mathrm{c}^{-}$ case of predissociation in the classification of Mulliken [17]. This is not a very favourable case for observing the $J$-variation of the Franck-Condon density : a simple argument is that the faster is the $v$-variation of the Franck-Condon density, the faster its $J$-variation should be. The rather slow $v$-variation of the predissociation rate results in a slow variation with $J$. However this $J$-variation remains important in our experiments for two reasons.

i) We have investigated very high $J$ values (frequently up to $J=140$ and even $J=174$ was reached once).

ii) The precision in some experiments is high; assuming that the Franck-Condon density does not depend on $J$ limits severely the accuracy of the interpretation of the data.

It is therefore necessary to evaluate the dependence of the Franck-Condon density with rotational quantum number $J$.

3.2 Calculation of the Franck-Condon denSITY. - 3.2.1 Principle. - The usual procedure is to numerically integrate the radial part of the Schrödinger equation for the discrete and the continuum states. Then the Franck-Condon density is evaluated by a numerical quadrature of the product of the two 


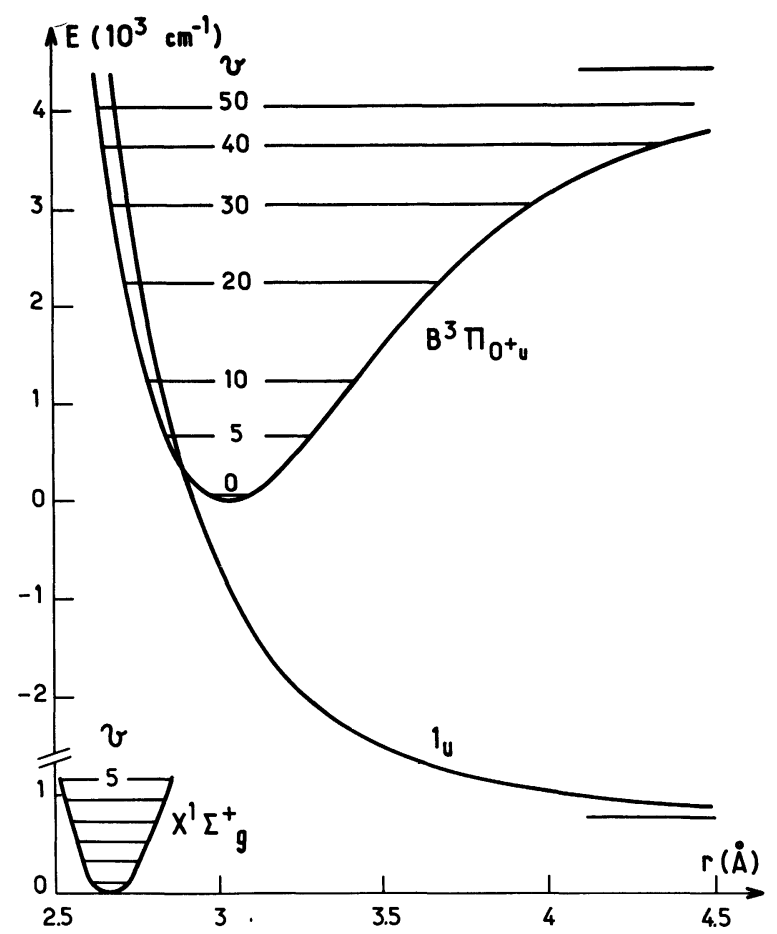

Fig. 1. - The $\mathrm{B}^{3} \Pi_{0^{+} \mathrm{u}}$ state potential curve (taken from Tellinghuisen [22]) and the $1_{u}$ state potential curve (taken from Chapman and Bunker [23]). The zero of energy is at the bottom of the B state well. The bottom of the $\mathrm{X}^{1} \Sigma_{\mathrm{g}}^{+}$well is also shown with a different zero of energy.

wavefunctions. We have preferred to use a semiclassical method. The principle of this method is to use the W.K.B. approximation of the wavefunction. The quadrature of the product of the two wavefunctions is then evaluated by the method of stationary phase. One then gets an equation giving the FranckCondon density as a function of various parameters depending of the two potential curves and a phase integral [18]. This method can be further improved by the use of the uniform approximation of the wavefunctions [19] instead of W.K.B. wavefunctions. We have used this latter formulation of the FranckCondon density [20].

The interest of the method derives from two facts :

- The physical meaning of the algebraic equation is quite clear [21];

- The calculations are much simpler than in the first method and therefore easier and cheaper to perform.

3.2.2 Results. - We have used for the B state a potential curve communicated to us by J. Tellinghuisen [22]. For the ${ }^{1} \Pi_{1}$ state, we have used two equations (units $\mathrm{cm}^{-1}, \AA$ ).

a) $V(r)=5.15 \times 10^{7} / r^{9}$ given by J. Tellinghuisen in reference [14].

b) $V(r)=\left(6.8 \times 10^{8} / r^{12}\right)+\left(9.3 \times 10^{5} / r^{6}\right)$ deduced by Chapman and Bunker [23] from the magnetic predissociation measurements (although the repulsive state was first assigned as a $0_{\mathrm{u}}^{-}$state, it is evidently [2] the curve of the same ${ }^{1} \Pi_{1 \mathrm{u}}$ state). For these two curves, the zero of energy is the dissociation limit of the ground state into two ${ }^{2} \mathrm{P}_{3 / 2}$ atoms; it can be easily related to the bottom of the B state potential well by knowledge of the $B$ state dissociation energy [24] and the ${ }^{2} P_{1 / 2}$ excitation energy [25].

We will use only the Franck-Condon density deduced from the potential curve $b$ as it gives a closer agreement with our experimental results [6]. Figure 1 shows the potential curve of the B state and the potential curve $b$ of the ${ }^{1} \Pi_{1 \mathrm{u}}$ state.

An estimate of the accuracy of the semiclassical approximation can be obtained by comparing our results to those of Chapman and Bunker [23]. However this comparison is significant only if we use exactly the same potential curve [38] for the B state. In figure 2, we have plotted, without any scale adjustment, two curves of the Franck-Condon density calculated by our method and the points corresponding to the values of Chapman and Bunker [23]. All calculations are made with $J=0$. The dotted curve is directly comparable to the points of reference [23] as it is calculated using the same B potential curve; the agreement is good, although some deviations do exist. The full curve is calculated with Tellinghuisen B state potential curve [22] and will be used from now on.

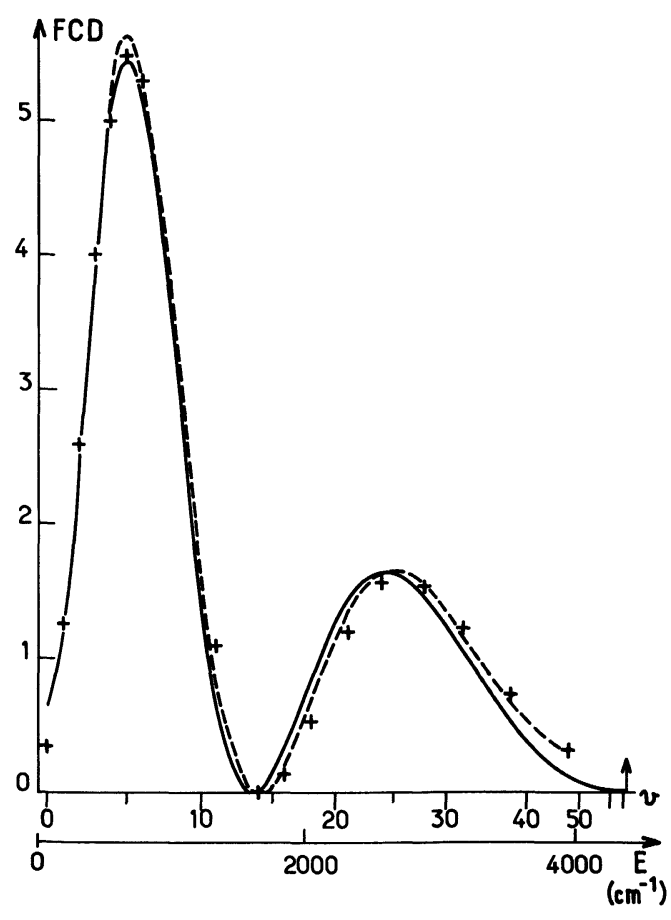

Fig. 2. - The calculated Franck-Condon density (in units of $10^{-4} \mathrm{~cm}$ ) as a function of the vibrational quantum number $v$ (the horizontal scale is linear in vibrational energy). The full and dotted curves represent the results of the semiclassical calculation, using either Tellinghuisen's [22] (full line) or reference [38] (dotted line) B state potential curve. This dotted curve is directly comparable to the values obtained in reference [23] and represented by the crosses. 
3.3 Variation with $J$ OF THE Franck-Condon DENSITY. - To study this variation, one must add the centrifugal term to both potentials and take into account the variation of energy of the levels under consideration (the spectroscopic constants of the B state are taken from reference [26]). The variation of the Franck-Condon density with $J$ is shown in figure 3 for some selected vibrational levels.

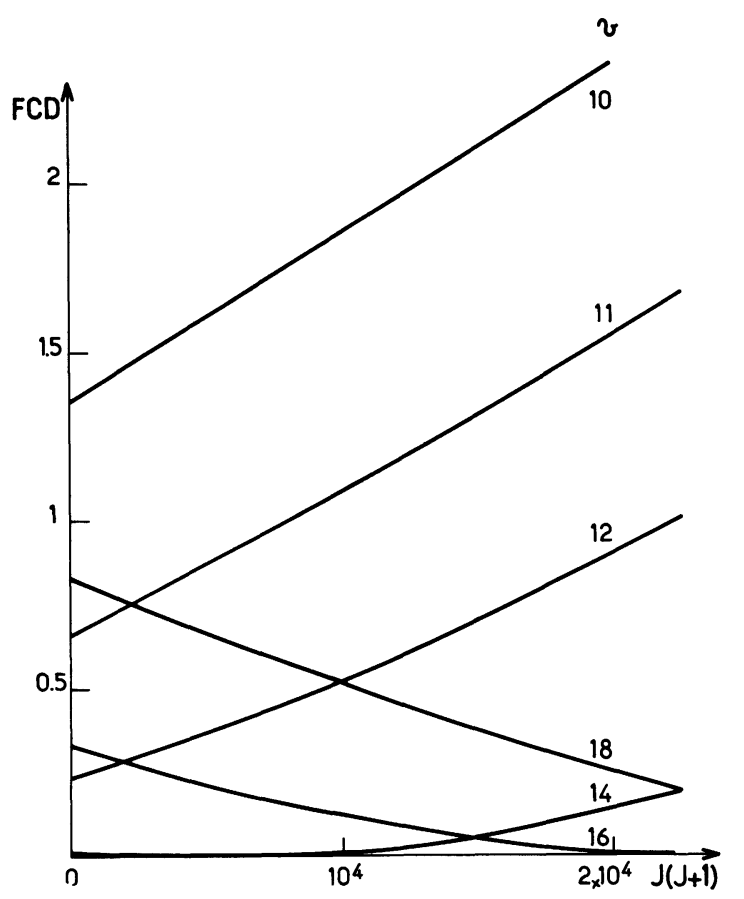

Fig. 3. - The curves represent the calculated Franck-Condon density (in units of $10^{-4} \mathrm{~cm}$ ) as a function of $J(J+1)$. Each curve is labelled by its vibrational quantum number.

Due to the $J(J+1)$ dependence of the centrifugal terms and of the energies, it appears clearly that a power series in $J(J+1)$ is adequate for approximating the observed behaviour :

$$
\begin{aligned}
F C D(v, J)=F C D(v, 0) & +P(v) J(J+1) \\
& +Q(v)(J(J+1))^{2}+\cdots
\end{aligned}
$$

In fact, in the range $J=0-150$, a very good representation of the calculated values of $F C D(v, J)$ can be obtained by fitting only the first three terms

$$
F C D(v, 0), \quad P(v), Q(v) .
$$

The relative deviation of the fit is smaller than $0.3 \%$ if $v$ is less than 30. We have plotted the curves representing the parameters $P(v)$ and $Q(v)$ in figure 4. It is important to note that $P(v)$ looks like $-\partial F C D / \partial v$ and $Q(v)$ like $\partial^{2} F C D / \partial v^{2}$. This type of relation between $P(v)$ and $\partial F C D / \partial v$ is in good agreement with the simple idea given in 3.1 , which relates the $J$ and $v$-variations of the Franck-Condon density; the minus sign which appears in the « relation » between $P(v)$ and $\partial F C D / \partial v$ has its origin in the fact that we

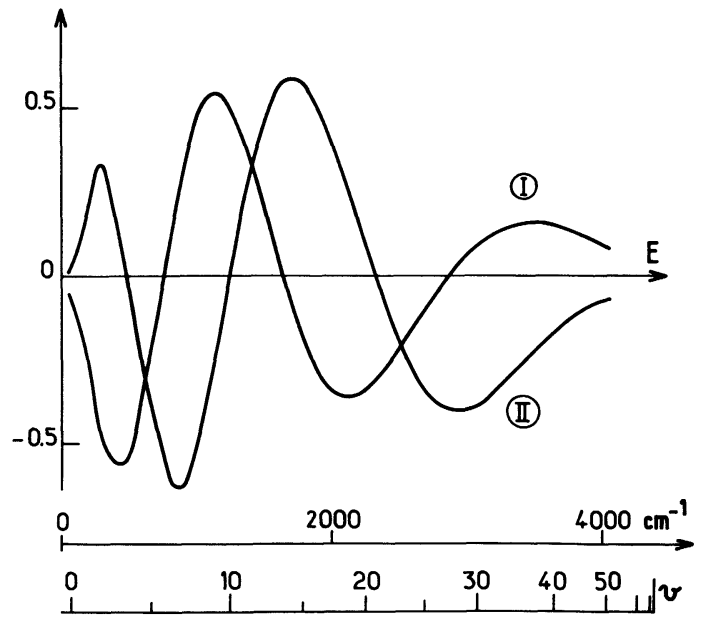

Fig. 4. - Curve I represents $P(v)$ (in units of $10^{-8} \mathrm{~cm}$ ) as a function of the vibrational energy. Curve II represents $Q(v)$ (in units of $10^{-13} \mathrm{~cm}$ ).

are studying a $\mathrm{c}^{-}$case ; a plus sign should be expected in $\mathrm{a} \mathrm{c}^{+}$case.

What we actually need is not the absolute value of the Franck-Condon density but its relative variation with $J$. We therefore write also :

$F C D(v, J)=$

$=F C D(v, 0)\left[1+p_{v} J(J+1)+q_{v}[J(J+1)]^{2}\right]$ with

$$
p_{v}=P(v) / F C D(v, 0) \quad q_{v}=Q(v) / F C D(v, 0) .
$$

These new quantities $p_{v}$ and $q_{v}$ have not been plotted because they diverge in the region where $F C D(v, 0)$ vanishes. We must note that all these parameters

$$
\left(F C D(v, 0), P(v), Q(v), p_{v}, q_{v}\right)
$$

are accurately known only if the knowledge of the ${ }^{1} \Pi_{1 \mathrm{u}}$ potential is sufficient and if the semiclassical calculation has introduced negligible errors. Although neither of these conditions is perfectly fulfilled, it appears $[6,21]$ that the $J$-variation of the FranckCondon density obtained by our semiclassical calculation using the potential curve $b$ is in very good agreement with the experimental determination of the same quantity, as will be demonstrated in the third paper of this series [27].

We are going to evaluate on each vibrational level the predissociation parameters for $J=0$, using for their $J$-variation equation (7) with the calculated $p_{v}$ and $q_{v}$ values. This procedure is not perfect; it would be more pleasant to measure all the predissociation parameters on each rovibrational level studied and to verify then that their $J$-variation is in agreement with theory. However this ideal procedure cannot be used because our data are usually not enough precise or complete.

4. Analysis of experimental data. - Most of the experiments analysed in the following pages have 
been described in detail in the theses of two of us (M. B. [28], J. V. [21]). The numerical analysis is, however, completely new. These experiments are of two types : lifetime measurements and fluorescence quantum yields in a molecular beam. Neither involves a magnetic field.

4.1 Time Resolved fluorescence deCAY. - These experiments were done with an apparatus described in references $[28,3,4]$. This apparatus permitted measurement of the fluorescence decay for single rovibrational levels of the $B$ state excited by a tunable pulsed dye laser. The decay curve is the sum of the exponential decays of all the hyperfine sublevels ( $n=15$ or 21 for para or ortho states). We can therefore represent the signal by

$$
J(t)=\sum_{\varepsilon F}(2 F+1) \mathrm{e}^{-\left[\Gamma_{\mathrm{rad}}+\Gamma_{\text {coll }}+\Gamma_{\mathrm{pred}}(v, J, \varepsilon, F)\right] t}
$$

where $\Gamma_{\text {coll }}$ is the collisional decay rate of the rovibrational level.

It is clear that such a decay curve is not exponential; however this character appears noticeably only for levels having a large predissociation probability. Before going further we must clarify some points : In order to simplify the calculation, and considering the large number of hyperfine sublevels, we have used an approximate form for $J(t)$ :

$$
J(t)=\sum_{I F}(2 F+1) \mathrm{e}^{-\left(\Gamma_{\mathrm{rad}}+\Gamma_{\mathrm{col1}}+\Gamma_{I . J F}\right) t},
$$

$\Gamma_{I J F}$ is deduced from (5) by introducing the $J$ dependence of the Franck-Condon density :

$$
\begin{aligned}
\Gamma_{I J F}=\left[C_{v}^{2} J(J+1)+\frac{a_{r}^{2}}{3}\left(\mathbf{I}^{2}+\frac{3(\mathbf{I} . \mathbf{J})^{2}+\frac{3}{2} \mathbf{I . J}-\mathbf{I}^{2} \mathbf{J}^{2}}{(2 J-1)(2 J+3)}\right)\right. & \left.-a_{v} C_{v} \sqrt{2} \mathbf{I} . \mathbf{J}\right] \\
& \times\left[1+p_{v} J(J+1)+q_{v}(J(J+1))^{2}\right] .
\end{aligned}
$$

From now on, when $a_{v}$ and $C_{v}$ will be used without any further specification, they will mean values of $a_{v J}$ and $C_{v J}$ for $J=0$.

4.1.1 Nonexponential decay curves. - These curves were recorded for the vibrational levels $v=7,8,9$, 10. For each curve, 20 points were measured and all curves recorded from one vibrational level were submitted to a least-squares fit. The theoretical expression fitted is given by equations (9) and (10). The parameters fitted are $\left(\Gamma_{\mathrm{rad}}+\Gamma_{\mathrm{col1}}\right), C_{r}^{2}$, and $a_{v}^{2}$ or the ratio $Y^{2}=a_{v}^{2} / C_{v}^{2}$. The product $a_{v} C_{v}$ was taken nega- tive as it has been shown to be by other experiments [5]. The $p_{v}$ and $q_{v}$ values were held constant at their calculated values. Table I gives the results obtained for the various parameters. The $\Gamma_{\text {coll }}$ term can be easily calculated, as the collision cross-section has been measured [3]. The pressure of iodine was $30 \mathrm{mtorr}$ in all the experiments, and the cell temperature was maintained at $25^{\circ} \mathrm{C}$. The values of the cross-sections appearing in reference [3] present some scatter; we replace these values by an average value

$$
\sigma=70 \pm 5 \AA^{2} .
$$

Table I. - Radiative decay rate and predissociation parameters deduced from analysis of the nonexponential decay curves for the vibrational levels $v=7,8,9,10$.

$\begin{array}{lcccc}v & 7 & 8 & 9 & 10 \\ \text { Number of levels } & 14 & 11 & 13 & 4 \\ p_{v} \times 10^{5} & 0.74 & 1.5 & 2.4 & 3.7 \\ q_{v} \times 10^{9} & -0.14 & -0.15 & - & 0.12 \\ \Gamma_{\text {rad }}\left(10^{6} \mathrm{~s}^{-1}\right) & 1.05 \pm 0.09 & 0.95 \pm 0.08 & 0.97 \pm 0.06 & 0.78 \pm 0.09 \\ C_{v}^{2}\left(\mathrm{~s}^{-1}\right) & 287 \pm 21 & 187 \pm 13 & 141 \pm 6 & 93 \pm 7 \\ a_{v}^{2}\left(10^{3} \mathrm{~s}^{-1}\right) & 324 \pm 26 & 232 \pm 18 & 143 \pm 11 & 112 \pm 17 \\ Y^{2} & 1130 \pm 100 & 1240 \pm 100 & 1010 \pm 65 & 1205 \pm 160\end{array}$

To illustrate the agreement between observed and calculated decays and also their strong nonexponential character, a logarithmic plot of the decay of the level $v=7, J=88$ is shown in figure $5 a$. In figure $5 b$, we have plotted the corresponding decay rates for the 21 hyperfine sublevels of this level. The very large differences between the decay rates of the levels $F=J+5$ and $F=J-5$ is due to the interference term in $a_{v} C_{v}$.

The quoted error bars are, in all cases, 3 standard deviations. It is interesting to note that $\Gamma_{\text {rad }}$ decreases as $v$ increases. Moreover the ratio $Y^{2}$, which should be independent of $v$ if one assumes perfect factorization of $a_{v}^{2}$ and $C_{v}^{2}$ into the product of an electronic matrix element by the Franck-Condon density, is reasonably constant. Finally, one must remark that for the levels close to $v=10$ there is a fast increase of $F C D(v, J)$ with $J$. As the determination of $C_{v}^{2}$ is mainly sensitive to high $J$ levels $\left(C_{v}\right.$ always appears through the product $C_{v} J$ ), the values determined if 


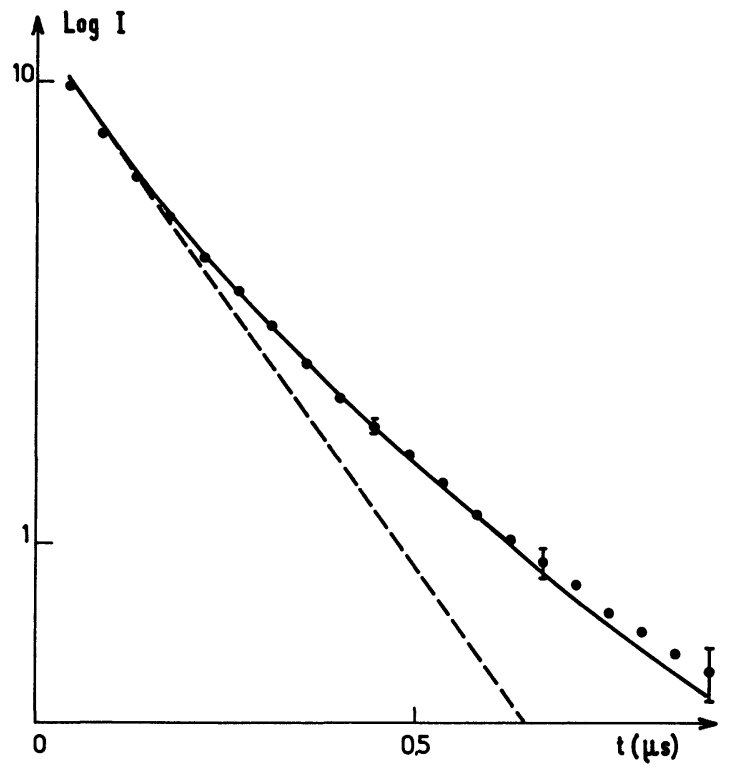

a)

Fig. 5a. - A logarithmic plot of the decay recorded for the level $v=7, J=88$. The points are experimental (some error bars are indicated); the full curve is the theoretical best fit. The straight dotted line has been plotted to illustrate the nonexponential nature of the decay.

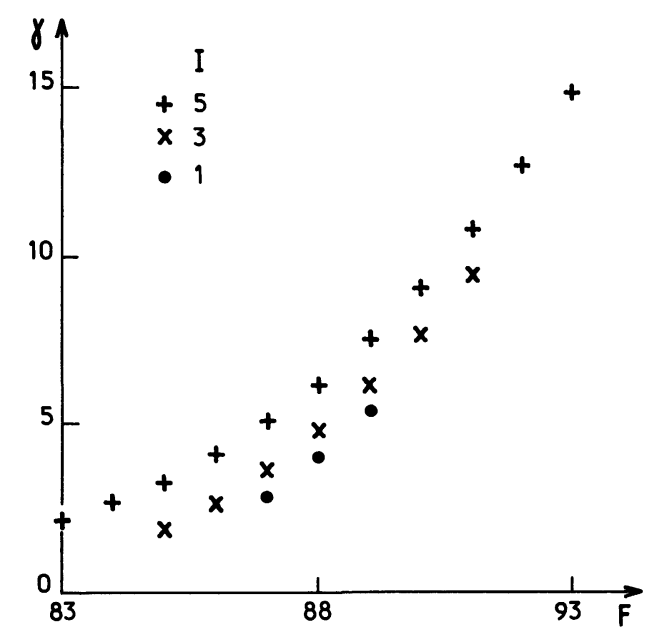

b)

Fig. $5 b$. - The values of $\gamma=\Gamma_{\mathrm{rad}}+\Gamma_{\text {coll }}+\Gamma_{I J F}$ (in units of $10^{6} \mathrm{~s}^{-1}$ ) used for the theoretical fit appearing in figure $5 a$ are plotted as a function of $F$ (horizontal scale) and $I$ (various symbols). The very large variation of $\gamma$ with $I$ and $F$ are evident and explain the strong nonexponential character of the decay.

one neglects the $J$ dependence of the $F C D$ differ very much from the values of table $I$; for instance in $v=10$, if one assumes $p_{v}=q_{v}=0$, the fitted value of $C_{v}^{2}$ is

$$
C_{10}^{2}=137 \pm 11 s^{-1}
$$

as compared to the value of table I

$$
C_{10}^{2}=93 \pm 7 \mathrm{~s}^{-1} \text {. }
$$

4.1.2 Lifetime measurements. - In most cases, when the predissociation probability is not too large, the deviation of the decay curve from an exponential curve is small and only a single lifetime can be deduced from experiments $[28,3]$. This is the case in the levels $v=11$ to 20 . Usually, the value given for the lifetime is an average from a study of the decay curve between the instant of the pulse and the time at which the, intensity is reduced by a factor $e^{2}$. Using equations (9) and (10), we can numerically calculate this average; we have submitted the experimental results to a least square fit procedure, the theoretical value being this average.

In most cases an alternation of theoretical lifetimes between ortho and para states should appear. This is easily observed for the levels with the strongest predissociation rates like $v=7$ or $8[4,28]$, but the predicted effect is of the order of the experimental accuracy for the levels $v=11-20$. Therefore the lifetime measurement is not directly sensitive to the value of $a_{v}^{2}$. In all cases, the value of $Y^{2}=a_{v}^{2} / C_{v}^{2}$ was taken from paragraph $4.2, Y^{2}=1120 \pm 160$. We have fitted $\Gamma_{\mathrm{rad}}$ and $C_{v}^{2}$ when the results were numerous enough; in other cases only $\Gamma_{\text {rad }}$ was determined, the values of $C_{v}^{2} / \Gamma_{\text {rad }}$ being taken from table III.

The results are given in table II. An asterisk designates that the quantity has not been fitted but taken from table III. The case of $v=18$ deserves a special comment : the observed decay rate is almost independent of $J[3]$; this is due to the fact that the FranckCondon density decreases with $J$ : therefore the hyperfine predissociation term decreases with $J$, while the gyroscopic predissociation term $C_{v J}^{2} J(J+1)$

Table II. - Values of the radiative decay rate $\Gamma_{\mathrm{rad}}$ and of the predissociation coefficient $C_{v}^{2}$ deduced from lifetime measurements for the vibrational levels $v=11$ 21. An asterisk near a value of $C_{v}^{2}$ means that, in this case, the ratio $C_{v}^{2} / \Gamma_{\mathrm{rad}}$ was held fixed at the value appearing in table III.

$\begin{array}{cccc}v & J \text { values } & \Gamma_{\text {rad }}\left(10^{6} \mathrm{~s}^{-1}\right) & C_{v}^{2}\left(\mathrm{~s}^{-1}\right) \\ \overline{11} & \overline{-} & \overline{-} & - \\ & 102,112,90 & 0.75 \pm 0.10 & 50 \pm 7 \\ 12 & 32,64,97 & 0.74 \pm 0.09 & 20 \pm 7 \\ 13 & 11,73 & 0.78 \pm 0.06 & 3 \pm 1.5 \\ 14 & 53 & 0.76 \pm 0.03 & 1 \pm 5^{*} \\ & & & 1 \\ 15 & 63 & 0.72 \pm 0.07 & 4 \pm 5^{*} \\ 16 & 57 & 0.73 \pm 0.07 & 15 \pm 12^{*} \\ 17 & 27 & 0.74 \pm 0.05 & 24 \pm 5^{*} \\ 18 & 37,58,85,104 & 0.78 \pm 0.06 & 32 \pm 6^{*} \\ 19 & 96 & 0.68 \pm 0.08 & 44 \pm 6^{*} \\ 20 & 40 & 0.73 \pm 0.10 & 54 \pm 11^{*} \\ 21 & 116\left({ }^{\mathrm{a}}\right) & 0.70 \pm 0.25 & 65 \pm 21^{*}\end{array}$

(a) Taken from reference [35]. 
increases with $J$, at least for low $J$ values. Therefore, although four levels have been studied, we have fitted only $\Gamma_{\mathrm{rad}} ; C_{v}^{2} / \Gamma_{\mathrm{rad}}$ and $Y^{2}$ being fixed.

Figure 6 gives a plot of the decay rate $\Gamma$ for the levels $v=11,12,13,18$ as a function of $J(J+1)$. This figure shows the agreement between obseryed and calculated values of $\Gamma$.

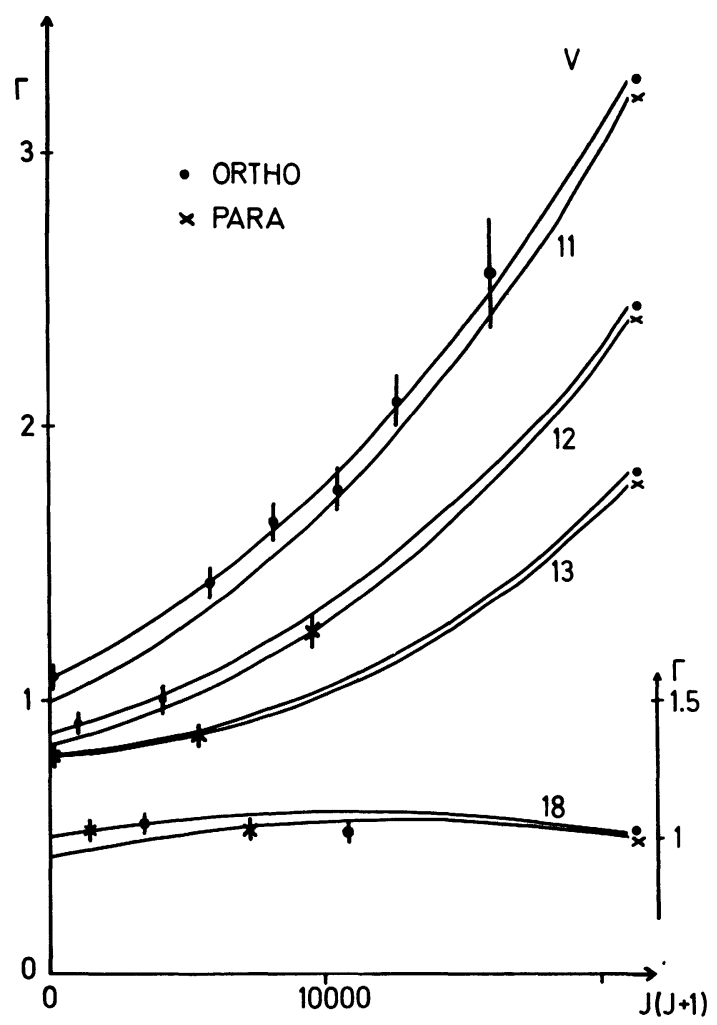

Fig. 6. - This figure presents the experimental results for $T$ (in units of $\left.10^{6} \mathrm{~s}^{-1}\right)$ as a function of $J(J+1)$ for the levels $v=11$, 12,13 and 18. The points are experimental (dots for ortho states and $\mathrm{X}$ for para states), and the curves are the theoretical best fit. The right vertical scale is for $v=18$ only.

\subsection{FLUORESCENCE QUANTUM YIELD IN A MOLECULAR} BEAM. - This experiment has been described earlier $[5,21]$ and was analysed then without the inclusion of the $J$ dependence of the Franck-Condon density. We recall briefly the principle : individual hyperfine sublevels of a rovibrational level of the $B$ state are excited selectively by a single-mode dye laser; the experiment is made on a molecular beam which, by reducing the Doppler effect, permits selective excitation. The intensity of the fluorescence light from the level $J \varepsilon F$ is given by :

$$
\mathfrak{J}(J, \varepsilon, F)=d \times J_{\mathrm{L}} \times \frac{2 F+1}{\Gamma_{\mathrm{rad}}+\Gamma_{\text {pred }}(v, J, \varepsilon, F)}
$$

where $d$ is a detection factor independent of $\varepsilon$ and $F$ and $J_{L}$ is the laser intensity, assumed sufficiently low to prevent saturation effects. The measurement of the fluorescence intensity has been restricted to the well resolved components of one triplet $F=J+5, J$, $J-5$ of ortho states. The two ratios

$$
J(F=J \pm 5) / J(F=J)
$$

have been measured and fitted to the theoretical expression (11). $\Gamma_{\text {pred }}(v, J, \varepsilon, F)$ is given by equation (4), $\Gamma_{I J F}$ being itself given by equation (10). The theoretical ratios $J(F=J \pm 5) / J(F=J)$ depend only on the quantities $C_{v}^{2} / \Gamma_{\mathrm{rad}}, a_{v}^{2} / \Gamma_{\mathrm{rad}}$, the sign of $a_{v} C_{v}$ and $p_{v}, q_{v}$. The coefficients $|\alpha(I \varepsilon J F)|^{2}$ are also necessary; they are obtained by diagonalization of the hyperfine Hamiltonian [36] using reasonable values of the hyperfine constants

$$
\left(e Q q^{\prime}=-500 \mathrm{MHz}, \quad C^{\prime}=30 \mathrm{kHz}\right) .
$$

From the data, the sign of $a_{v} C_{v}$ appears to be negative. In the least-squares procedure, only $a_{v}^{2} / \Gamma_{\mathrm{rad}}$ and $C_{v}^{2} / \Gamma_{\mathrm{rad}}$ are fitted (or $C_{v}^{2} / \Gamma_{\mathrm{rad}}$ and $Y^{2}=a_{v}^{2} / C_{v}^{2}$ ). Table III gives the results for $a_{v}^{2} / \Gamma_{\mathrm{rad}}, C_{v}^{2} / \Gamma_{\mathrm{rad}}$, and $Y^{2}$. This last quantity is not given when its error bar is too large. The error bar is taken equal to 3 standard deviations and appears in most cases to be rather large (approximately $30 \%$ ). In the case $v^{\prime}=9$, only one level was studied, $\left(J^{\prime}=20\right)$. The data, on this low $J$ level, is only sensitive to $a_{v}^{2} / \Gamma_{\mathrm{rad}}$; the value of $C_{v}^{2} / \Gamma_{\mathrm{rad}}$ was held fixed at the value calculated from table $I$.

Table III. - Values of $C_{v}^{2} / \Gamma_{\mathrm{rad}}, a_{v}^{2} / \Gamma_{\mathrm{rad}}$ and $Y^{2}=a_{v}^{2} / C_{v}^{2}$ deduced from the fluorescence intensity measurements on a molecular beam.

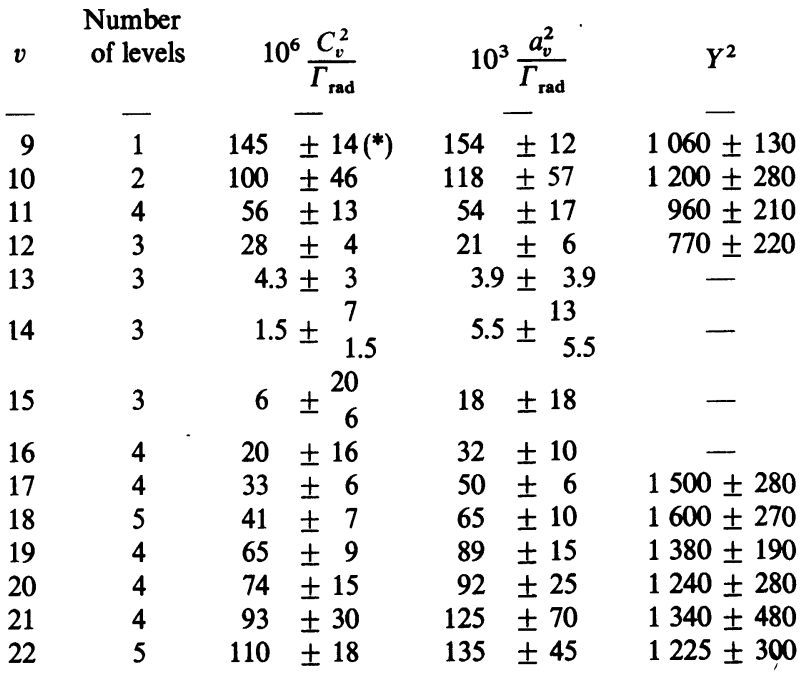

$\left(^{*}\right)$ Fixed (from table I).

In order to illustrate the quality of the fits, we present, in figure 7, a plot of the experimental and theoretical ratios $\mathrm{J}(F=J \pm 5) / J(F=J)$ for the level $v=22$. The curves correspond to the best theoretical fit.

From this rather large set of levels, it appears clearly that $a_{v}^{2}$ and $C_{v}^{2}$ have the right vibrational dependence, as $\Gamma_{\text {rad }}$ varies slowly with $v$ (see table II). The ratio $Y^{2}$ is not far from constant. However it is 


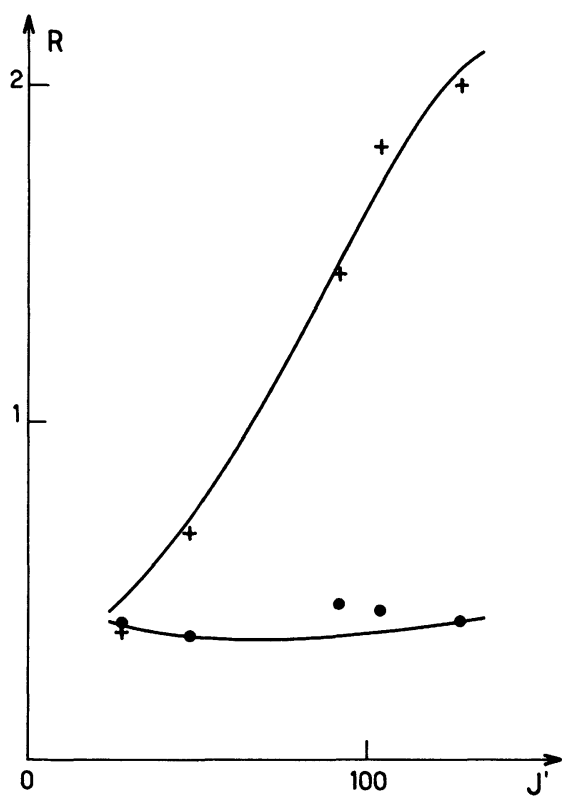

Fig. 7. - The values of the ratio $R=\mathfrak{J}(F=J+5) / \mathfrak{J}(F=J)(\bullet)$ and $R=J(F=J-5) / J(F=J)(+)$ are plotted as a function of $J^{\prime}$ for the level $v^{\prime}=22$. The full line curves represent the best fit to the data.

not excluded that $C_{v}^{2}$ vanishes at a slightly higher vibrational level than does $a_{v}^{2}$. This would explain the tendency of $Y^{2}$ to be smaller than its average for $v<14$ and larger for $v>14$. This phenomenon would be easily explained as a small breakdown of the Franck-Condon approximation (i.e. the approximation which says that $a_{v}^{2}, C_{v}^{2}$ are both strictly proportional to the same $F C D$ which would be valid only if the electronic matrix elements involved have no dependence on internuclear distance).

By taking into account all data of tables I and III, it is possible to evaluate a mean value of $Y^{2}$. Using weights inversely proportional to the square of the error bars, we get :

$$
Y^{2}=1120 \pm 160,
$$

the uncertainty in this case being equal to the root mean square deviation of the $Y$ values. This is the value used for the calculation of table II.

4.3 OTHER EXPERIMENTS. - 4.3.1 Lifetime measurements for selectively excited hyperfine sublevels. We have performed this experiment on one rotational level $v^{\prime}=19, J^{\prime}=84$, excited in the molecular beam [21]. The lifetime was measured by the phase shift method for the three hyperfine sublevels $F=J+5$, $J, J-5$ which were well resolved in our apparatus. The values of the lifetimes $\tau$ appear in table IV. We give also the fluorescence intensities $\mathfrak{J}$ in arbitrary units for the same sublevels. As required by equation (11), the ratio $\rho=J /(2 F+1) \tau$ should be constant. This constancy is not incompatible with the results, but the apparent variation of $\rho$ may be due to an error made in the determination of the phase of the excitation modulation. This error seems very probable in our experiment (see details in [21]), and explains the monotonic variation of $\rho$ with respect to $\tau$. Therefore the lifetime data, though corroborating our quantum yield experiments, do not give any really new and reliable information.

Table IV. - Lifetime $\tau$, fluorescence intensities $\mathfrak{J}$, and values of the ratio $\rho=J /(2 F+1) \tau$ for the sublevels $F=J-5, J, J+5$ of the level $v=19, J=84$.

$\begin{array}{cccc}F & 79 & 84 & 89 \\ \tau(\mu \mathrm{s}) & 0.81 \pm 0.07 & 0.55 \pm 0.07 & 0.15 \pm 0.05 \\ J & 66 \pm 5 & 52 \pm 5 & 21 \pm 3 \\ \rho & 0.52 \pm 0.10 & 0.56 \pm 0.12 & 0.78 \pm 0.25\end{array}$

4.3.2 Experiments of Ducasse and Couillaud. These experiments [29] involved the technique of saturated absorption spectroscopy. Their apparatus gave a linewidth of the order of one $\mathrm{MHz}$. This was just insufficient to observe linewidth variations from one hyperfine component to another, as the natural width, including predissociation, is almost always less than $1 \mathrm{MHz}$.

Ducasse and Couillaud have proved that, in the limit of low saturation, the signal from one hyperfine component should be proportional to the quantity $(2 F+1) /\left(\Gamma_{\text {rad }}+\Gamma_{\text {pred }}(v, J, \varepsilon, F)\right)$. This means that their experiment should give the same information as our experiment of fluorescence in the molecular beam. Indeed, the same type of signal was observed but it was not possible [29] to deduce, from their experiments, values of the $a_{v}^{2} / \Gamma_{\mathrm{rad}}$ and $C_{v}^{2} / \Gamma_{\mathrm{rad}}$ in agreement with our determinations. It seems that the most probable explanation of this discrepancy is due to the difficulty of accurately controlling the saturation parameter.

4.3.3 Experiments on the levels $v^{\prime}=43, J^{\prime}=12$ and 16 excited by the $5145 \AA$ line of the $\mathrm{Ar}^{+}$laser. Two types of experiments have been done. One involved fluorescence excitation in a molecular beam, made by Ezekiel et al. [30]. This experiment, analogous to our own, was made in 1975 and was in fact the first evidence of a hyperfine predissociation. It was evidently impossible to understand it at that time. It is now easy to interpret the anomalous ratio of intensities of hyperfine components in terms of the predissociation parameters. We have measured the ratio $J(F=J \pm 5) / J(F=J)$ for the two levels $J^{\prime}=12$ and 16 from the experimental curve given in reference [30]. These quantities are really sensitive only to $a_{v}^{2} / \Gamma_{\text {rad }}$; assuming that $Y^{2}=1120 \pm 160$, we obtain

$$
\frac{a_{43}^{2}}{\Gamma_{\mathrm{rad}}}=(35 \pm 12) \cdot 10^{-3} .
$$

The other experiment is the saturated absorption spectroscopy of Bordé, Camy and Decomps [31]. The intensities of some hyperfine components are clearly not in agreement with a calculation neglecting hyperfine predissociation, but no quantitative result 
for the predissociation parameters is presently available.

4.3.4 Experiment of Brand, Schulz and Steudel. This experiment [32] is exactly analogous to our fluorescence study on a molecular beam. The levels studied were four rotational levels of the vibrational state $v^{\prime}=18$. The resolution of the experiment permits an analysis of all hyperfine components. Their analysis, made without inclusion of the $J$ dependence of the Franck-Condon density, gives values which are presented in table V. The comparison with our values deduced from table III for the same parameters is not satisfactory. We see no reason which explains this discrepancy.

Table V. - Comparison between the results of Brand, Schulz and Steudel [32] and our results for the level $v=18$.

$$
\begin{array}{lcc} 
& \text { Reference [32] } & \text { Our results } \\
\frac{\left|C_{18}\right|}{\sqrt{\Gamma_{\mathrm{rad}}}} & (9.8 \pm 4) \cdot 10^{-3} & (6.4 \pm 0.5) \cdot 10^{-3} \\
\frac{\left|a_{18}\right|}{\sqrt{\Gamma_{\mathrm{rad}}}} & (138 \pm 60) .10^{-3} & (255 \pm 20) \cdot 10^{-3}
\end{array}
$$

4.3.5 The experiment of Man, Cerez and Brillet. Very recently this group has communicated a direct observation of the hyperfine predissociation through linewidth measurements in saturated absorption spectroscopy [33]. This was obtained with the very narrow linewidth $(100 \mathrm{kHz})$ of their apparatus, using a $\mathrm{He}-\mathrm{Ne}$ laser at $612 \mathrm{~nm}$. From their experiments they have calculated the predissociation parameters for the level $v=9, J=48$. Table VI gives the comparison between their values and our own obtained by correcting the data of table $I$ of this paper for the $J$ dependence. The agreement is reasonable, considering that the present experiment [33] is extremely difficult.

Table VI. - Comparison between the results of Man, Cerez and Brillet and our determination of the predissociation parameters for the level $v=9, J=48$.

$$
\begin{array}{lcc} 
& \text { Reference [33] } & \text { Our values } \\
a_{v J}^{2} & (224 \pm 70) .10^{3} \mathrm{~s}^{-1} & - \\
C_{v J}^{2} & (137 \pm 60) \mathrm{s}^{-1} & (149 \pm 6) \mathrm{s}^{-1}
\end{array}
$$

5. Fano profile for iodine absorption line? - This type of effect appears when absorption towards a discrete state coupled to a continuum and absorption towards the continuum are both allowed; it gives an asymmetric lineshape [34]. It has been observed usually in cases where the natural linewidth was larger than the Doppler width. Evidently, in iodine one should eliminate the Doppler effect to try to observe this asymmetric profile.
As the absorption from the ground state towards both the $\mathrm{B}^{3} \Pi_{0+\mathrm{u}}$ and the ${ }^{1} \Pi_{1 \mathrm{u}}$ states is allowed, the effect should exist, but, as we will see, the expected asymmetry is very small. As we want only an order of magnitude estimate, let us neglect the nuclear spins. Figure 8 shows the different transitions connecting the ground state $\mathrm{X}^{1} \Sigma_{\mathrm{g}}^{+} v^{\prime \prime} J^{\prime \prime}$ to the various levels of the B and $1_{u}$ states. Obviously, the level $v^{\prime \prime} J^{\prime \prime}$ is coupled to the ${ }^{1} \Pi_{1 \mathrm{u}}$ level $J^{\prime}=J^{\prime \prime}+1$ either directly through an $\mathrm{R}$ line or indirectly by absorption to the $\mathrm{B}^{3} \Pi_{0^{+} \mathrm{u}}$ level $J^{\prime}=J^{\prime \prime}+1$ and then by predissociation. This is the prerequisite for the existence of a Fano effect.

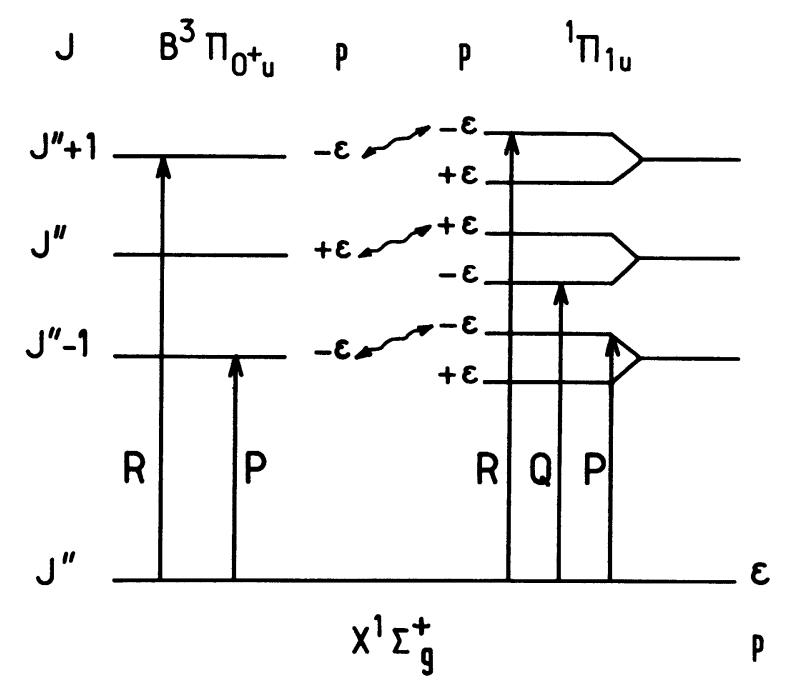

Fig. 8. - Possible transitions from the ground state $\mathrm{X}^{1} \Sigma_{\mathrm{g}}^{+}$to the $\mathrm{B}^{3} \Pi_{0+\mathrm{u}}$ and the ${ }^{1} \Pi_{1 \mathrm{u}}$ states. The total parity $p$ of each level is indicated $\left(\varepsilon=(-1)^{J^{\prime \prime}}\right)$. The predissociation coupling $(\leftarrow m \rightarrow)$ occurs only between states of the same total parity.

The $q$ parameter, defined by Fano, gives the asymmetry of the line profile : the ratio of dispersion to absorption in the lineshape is given by $\frac{2 q}{q^{2}-1} \approx \frac{2}{q}$ for large $q . q^{2}$ is given by [34]

$$
q^{2}=\frac{4}{\pi \hbar \Gamma_{\text {pred }}} \frac{\left|\left\langle\mathrm{B} v^{\prime} J^{\prime}|D| \mathrm{X}^{1} \Sigma_{\mathrm{g}}^{+} v^{\prime \prime} J^{\prime \prime}\right\rangle\right|^{2}}{\left|\left\langle{ }^{1} \Pi_{1 \mathrm{u}} E J^{\prime}|D| \mathrm{X}^{1} \Sigma_{\mathrm{g}}^{+} v^{\prime \prime} J^{\prime \prime}\right\rangle\right|^{2}},
$$

where $D$ is the electric dipole operator. Using the Hönl London $S$ coefficient, we write :

$$
\begin{aligned}
q^{2}=\frac{4}{\pi \hbar \Gamma_{\text {pred }}} \frac{S_{J^{\prime} J^{\prime \prime}}\left(0_{\mathrm{u}}^{+}-0_{\mathrm{g}}^{+}\right)}{S_{J^{\prime} J^{\prime \prime}}\left(1_{\mathrm{u}}-0_{\mathrm{g}}^{+}\right)} \times \\
\quad \times \frac{\left|D_{\mathrm{BX}}\right|^{2}}{\left|D_{1_{\mathrm{u}} \mathrm{X}}\right|^{2}} \cdot\left|\frac{\left\langle\mathrm{B} v^{\prime} \mid \mathrm{X} v^{\prime \prime}\right\rangle}{\left\langle 1_{\mathrm{u}} E \mid \mathrm{X} v^{\prime \prime}\right\rangle}\right|^{2} .
\end{aligned}
$$


In the high $J$ limit

$$
\begin{aligned}
S_{J^{\prime} J^{\prime \prime}}\left(0_{\mathrm{u}}^{+}-0_{\mathrm{g}}^{+}\right)= & \frac{1}{2} \text { for } \mathrm{R} \text { and } \mathrm{P} \text { lines } ; \\
S_{J^{\prime} J^{\prime \prime}}\left(1_{\mathrm{u}}-0_{\mathrm{g}}^{+}\right)= & \frac{1}{4} \text { for } \mathrm{R} \text { and } \mathrm{P} \text { lines }, \\
& \frac{1}{2} \text { for } \mathrm{Q} \text { lines } .
\end{aligned}
$$

From the work of Tellinghuisen [13], the ratio of the electronic matrix elements of the dipole is easily calculated

$$
\left(\frac{D_{\mathrm{BX}}}{D_{1_{\mathrm{u}} \mathrm{x}}}\right)^{2} \approx 6
$$

Finally, the $B$ and $1_{u}$ potential curves are almost linear and parallel $\left(V_{\mathrm{B}}(r) \simeq V_{1 \mathrm{u}}(r)-\delta, \delta \simeq 1000 \mathrm{~cm}^{-1}\right)$ in the region of internuclear distance accessible from the first vibrational levels of the ground state (see figure 1 ; the ground state potential curve is taken from reference [37]). Then it is possible to use the principle of reflection to write :

$$
\begin{aligned}
\left|\left\langle\mathrm{B} v^{\prime} \mid \mathrm{X} v^{\prime \prime}\right\rangle\right|^{2} \approx \hbar \omega_{v^{\prime}} \mid\left\langle 1_{\mathrm{u}^{\prime}}\right. & E= \\
= & E\left(v^{\prime}\right)+\left.\delta\left|\mathrm{X} v^{\prime \prime}\right\rangle\right|^{2} .
\end{aligned}
$$

The factor $\hbar \omega_{v^{\prime}}$ originates from the different normalizations of the wavefunctions of bound state and continuum states [21]. Then one gets approximately, by taking $\omega_{v^{\prime}} \approx 100 \mathrm{~cm}^{-1}$ and $\Gamma_{\text {pred }} \approx 10^{6} \mathrm{~s}^{-1}$

$$
q^{2}=10^{8} \frac{\left|\left\langle 1_{\mathrm{u}}, E=E\left(v^{\prime}\right)+\delta \mid \mathrm{X} v^{\prime \prime}\right\rangle\right|^{2}}{\left|\left\langle 1_{\mathrm{u}}, E=E\left(v^{\prime}\right) \mid \mathrm{X} v^{\prime \prime}\right\rangle\right|^{2}} .
$$

Usually, the ratio of Franck-Condon densities is not far from unity; then $q$ will be of the order of $10^{4}$ leading to a line asymmetry of $10^{-4}$. However, if the upper Franck-Condon factor is very small, $q$ could be much smaller than this value of $10^{4}$; this means that one should look for a transition which is almost forbidden because of a very low B-X Franck-Condon factor. Such transitions exist, but should be very difficult to study.

6. Conclusion. - This paper has been devoted to the natural predissociation of the $B$ state of $\mathrm{I}_{2}$, without introducing the magnetic predissociation. Magnetic effects will be discussed in the next paper of this series. As new values of the gyroscopic parameter $C_{v}^{2}$ will be presented there, it is too early to comment in detail on the numerical results obtained here. However, it is possible to say that the picture of the gyroscopic and hyperfine predissociation of the B state is firmly established and that all the experiments described are well represented by the simplifyed version of the predissociation rate (equations (4) and (5)). Moreover, the ratio of the hyperfine to gyroscopic predissociation parameters is now rather accurately known and its value is reasonably constant as is predicted by theory. Finally, introduction of the $J$ dependence of the Franck-Condon density has given a significant improvement in the interpretation of the data.
[1] Broyer, M., Vigué, J. and Lehmann, J. C., Chem. Phys. Lett. 22 (1973) 313.

Broyer, M. and Vigué, J., Colloque international du C.N.R.S. no $^{\circ}$ 217, Aussois (1973) p. 185.

Vigué, J., Broyer, M. and LehmanN, J. C., J. Phys. B 7 (1974) L158.

[2] Vigué, J., Broyer, M. and Lehmann, J. C., J. Chem. Phys. 62 (1975) 4941

[3] Broyer, M., Vigué, J. and Lehmann, J. C., J. Chem. Phys. 63 (1975) 5428

[4] Broyer, M., Vigué, J. and Lehmann, J. C., J. Chem. Phys. 64 (1976) 4793

[5] Vigué, J., Broyer, M. and Lehmann, J. C., J. Phys. B 10 (1977) L379.

[6] Vigué, J. and Broyer, M., Colloque international du C.N.R.S. $\mathrm{n}^{\circ}$ 273, Aussois (1977) p. 333.

[7] Chutjian, A., Link, J. K. and Brewer, L., J. Chem. Phys. 46 (1967) 2666

[8] Chutjian, A. and James, T. C., J. Chem. Phys. 51 (1969) 1242.

[9] Chutjian, A., J. Chem. Phys. 51 (1969) 5414.

[10] Wasserman, E., Falconer, W. E. and Yager, W. A., J. Chem. Phys. 49 (1968) 1971.

[11] Busch, G. E., Mahoney, R. T., MoRse, R. I. and Wilson, K. R., J. Chem. Phys. 51 (1969) 837.

[12] Brewer, L. and Tellinghuisen, J., J. Chem. Phys. 56 (1972) 3929.
[13] Tellinghuisen, J., J. Chem. Phys. 58 (1973) 2821.

[14] Tellinghuisen, J., J. Chem. Phys. 57 (1972) 2397.

[15] Vigute, J., Broyer, M. and LehmanN, J. C., J. Physique (First article of this series).

[16] Czarny, J., Felenbok, P. and Lefebvre-Brion, H., J. Phys. $B 4$ (1971) 124.

[17] Mulliken, R. S., J. Chem. Phys. 33 (1960) 247.

[18] CHILD, M. S., J. Mol. Spectrosc. 33 (1970) 487.

[19] Miller, W. H., J. Chem. Phys. 48 (1968) 464.

[20] Child, M. S., Can. J. Phys. 53 (1975) 1838.

[21] VIGUÉ, J., Thesis, Paris (1978), unpublished.

[22] Tellinghuisen, J., Private Communication (1976).

[23] Chapman, G. D. and Bunker, P. R., J. Chem. Phys. 57 (1972) 2951.

[24] YeE, K. K., Chem. Phys. Lett. 21 (1978) 334.

[25] MoOre, C., Atomic Energy Levels III, N.B.S.

[26] Wei, J. and Tellinghuisen, J., J. Mol. Spectrosc. 50 (1974) 317

[27] Vigué, J., Broyer, M. and Lehmann, J. C., J. Phisique (3rd article of this series).

[28] Broyer, M., Thesis, Paris (1977), unpublished.

[29] Couillaud, B. and Ducasse, A., Opt. Commun. 21 (1977) 199. Couillaud, B. and Ducasse, A., Thesis, Université de Bordeaux (1978).

[30] Hackel, L. A., Casleton, K. H., Kukolich, S. G. and EzeKIEL, S., Phys. Rev. Lett. 35 (1975) 568. 
[31] Borde, J., Camy, G. and Decomps, B., Phys. Rev. A20 (1979) 254.

CAMY, G., Thesis, University P. and M. Curie, Paris (1979) [32] Brand, H., Schulz, H. H. and Steudel, A., Phys. Lett. 63 A (1977) 235

[33] Man, C., Cerez, P. and Brillet, A., 11th EGAS Conference, - Paris (1979).

[34] Fano, U., Phys. Rev. 124 (1961) 1866.
[35] Paisner, J. A. and Wallenstein, R., J. Chem. Phys. 61 (1974) 4317.

[36] Levenson, M. D. and Schawlow, A. L., Phys. Rev. A6 (1972) 10.

[37] Leroy, R. J., J. Chem. Phys. 52 (1970) 2683.

[38] Steinfeld, J. I., ZARE, R. N., Jones, L., LeSK, M. and KLEMPERER, W., J. Chem. Phys. 42 (1965) 25. 